\begin{tabular}{|c|l|}
\hline Title & Physarum solver : A biologically inspired method of road-network navigation \\
\hline Author(s) & Tero, A tsushi; Kobay ashi, Ryo; Nakagaki, Toshiyuki \\
\hline Citation & $\begin{array}{l}\text { PhysicaA Statistical Mechanics and its A pplications, 363(1), 115-119 } \\
\text { https://doi.org/L0.1016/.physa.2006.01.053 }\end{array}$ \\
\hline Issue Date & 2006-0415 \\
\hline Doc URL & http://hdl.handle.net/2115/28042 \\
\hline Type & article(author version) \\
\hline Note(URL) & http://www.elsevier.com/wps/find/ournaldescription.cws_home/505702/description \\
\hline File Information & PA SMA 363-1.pdf \\
\hline
\end{tabular}

Instructions for use 


\title{
Physarum solver: \\ - a biologically inspired method of road-network navigation -
}

\author{
Atsushi Tero ${ }^{\mathrm{a}}$, Ryo Kobayashi ${ }^{\mathrm{a}}$, Toshiyuki Nakagaki ${ }^{\mathrm{b}}$ \\ a Department of Mathematical and Life Sciences, \\ Hiroshima University, Higashi-Hiroshima 739-8626, Japan \\ b Research Institute for Electronic Science, \\ Hokkaido University, Sapporo, 060-0812, Japan \\ Creative Research Initiative (SOUSEI), \\ Hokkaido University, Sapporo, 001-0021, Japan
}

\begin{abstract}
We have proposed a mathematical model for the adaptive dynamics of the transport network in an amoeba-like organism, the true slime mold Physarum polycephalum. The model is based on physiological observations of this species, but can also be used for path-finding in the complicated networks of mazes and road maps. In this paper, we describe the physiological basis and the formulation of the model, as well as the results of simulations of some complicated networks. The path-finding method used by Physarum is a good example of cellular computation.
\end{abstract}

Key words: amoeboid movement, navigation algorithm, Physarum polycephalum, path-finding

PACS: 87.17.Aa, 89.75.Hc, 89.65.Lm, 89.40.-a,

\section{Introduction}

Technologies capable of navigating complex networks such as road traffic or Internet systems are fundamental to modern society. The most popular technique for solving shortest path problems is Dijkstra's algorithm, which is based on combinatorial optimization. Although this method is capable of correctly identifying the shortest route, its disadvantage lies in the fact that with an increasing number of nodes the required computational time becomes excessive. To overcome this difficulty, two biologically-inspired methods, genetic algorithm and ant algorithm, have been proposed[1]. However, neither method is 
guaranteed to find the shortest route, as both are based on stochastic processes.

Another requirement for navigation algorithms is that of flexible adaptability and re-routing, for example during traffic congestion or following accidents. The challenge is therefore to develop a method that meets all three requirements of swiftly determining all possible paths, finding the shortest route, and adaptable re-routing. A completely new method that fulfills these criteria is demonstrated using a mathematical path-finding model derived from observation of an amoeboid organism, the true slime mold.

The plasmodium of true slime mold Physarum polycephalum can tackle a maze and some other types of geometrical puzzle, and can successfully optimize survival tasks[2-6]. The challenge is to extract a mathematical algorithm for this natural computation.

The body of the plasmodium contains a network of tubes, which enables nutrients and chemical signals to circulate through the organism. When food sources are presented to a starved plasmodium that has spread over the entire surface of an agar plate, parts of the organism concentrate over the food sources and are connected by only a few tubes. The path connecting these parts of the plasmodium is the shortest possible, even in a maze $[2,4]$.

The physiological mechanism of tube formation has been established: tubes thicken in a given direction when shuttle streaming of the protoplasm persists in that direction for a certain time [7]. This implies positive feedback between flux and tube thickness, as the conductance of the sol is greater in a thicker channel. Here we propose a mathematical model for this mechanism.

Past experiments have revealed two empirical rules describing the changes in the tubular structure of the plasmodium: first, open-ended tubes are likely to disappear; and second, when two or more tubes connect the same two food sources, the longer tube tends to disappear [4]. The mathematical model mentioned above has to reproduce these phenomenological rules. After discussing the validity of the model, we apply it to the navigation problems posed by a complicated road map or large labyrinth.

\section{Mathematical modeling of maze-solving by Physarum}

Let us describe the nature of the model using the maze illustrated in Fig. 1. This set-up was used in a maze-solving experiment with Physarum [2]. Each segment in the diagram represents a section of tube. Two special nodes, corresponding to the food sources, are named $N_{1}$ and $N_{2}$, and the other nodes 
are denoted as $N_{3}, N_{4}, N_{5}$, and so forth. One of the food source nodes $\left(N_{1}\right)$ always acts as a source and the other $\left(N_{2}\right)$ acts as a sink. The section of tube between $N_{i}$ and $N_{j}$ is denoted as $M_{i j}$. If several tubes connect the same pair of nodes, we place nodes in the centre of the tubes to guarantee the uniqueness of the connecting segments.

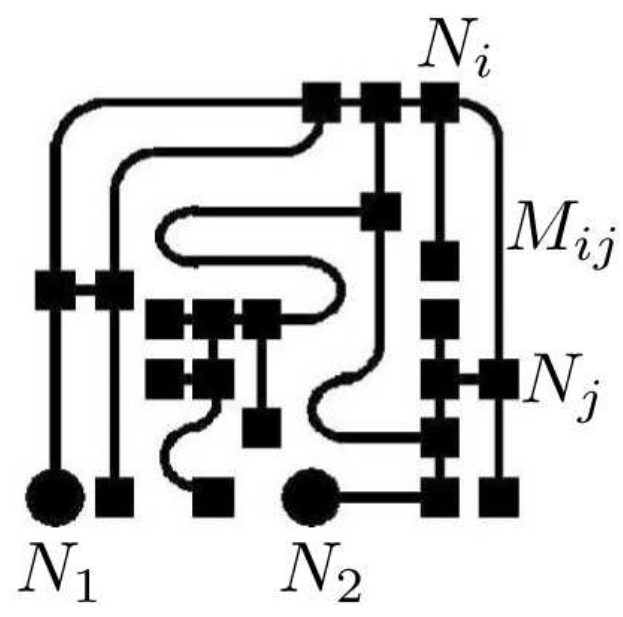

Fig. 1. Expression of the maze by graph. Ordinary nodes are indicated by squares and the nodes corresponding the food sources are given by circles.

The variable $Q_{i j}$ is the flux through $M_{i j}$ from $N_{i}$ to $N_{j}$. Assuming an approximately Poiseuille flow, the flux $Q_{i j}$ is given by:

$$
Q_{i j}=\frac{D_{i j}}{L_{i j}}\left(p_{i}-p_{j}\right)
$$

where $p_{i}$ is a pressure at the node $N_{i}, L_{i j}$ is a length of the segment $M_{i j}$ and $D_{i j}$ is its conductivity (the reciprocal of its resistance per unit length). By considering Kirchhoff's law at each node, we have:

$$
\sum_{i} Q_{i j}=0 \quad(j \neq 1,2) .
$$

For the source node $N_{1}$ and the sink node $N_{2}$, the following two equations hold:

$$
\sum_{i} Q_{i 1}+I_{0}=0, \quad \sum_{i} Q_{i 2}-I_{0}=0
$$

where $I_{0}$ is the flux from the source node. It should be noted that $I_{0}$ is a constant in our model.

In order to describe the adaptation of tubular thickness, we assume that the conductivity $D_{i j}$ changes in time according to the flux $Q_{i j}$ as follows: 


$$
\frac{d}{d t} D_{i j}=f\left(\left|Q_{i j}\right|\right)-r D_{i j}
$$

where $f(Q)$ is a increasing function with $f(0)=0$. This equation implies that conductivity tends to decline exponentially, but is enhanced by the flux through the segment. In this article, we adopt the functional form $f(Q)=$ $\alpha|Q|$. Hence, the adaptation equation is simply expressed by:

$$
\frac{d}{d t} D_{i j}=\alpha\left|Q_{i j}\right|-r D_{i j}
$$

Note that the edge length $L_{i j}$ 's are kept constant throughout the adaptation process. The network Poisson equation for the pressure is derived from the equations (1), (2), and (3) as follows:

$$
\sum_{i} \frac{D_{i j}}{L_{i j}}\left(p_{i}-p_{j}\right)=\left\{\begin{array}{cc}
-I_{0} & \text { for } j=1 \\
I_{0} & \text { for } j=2 \\
0 & \text { otherwise }
\end{array}\right.
$$

By setting $p_{2}=0$ as a basic pressure level, all $p_{i}$ 's can be determined by solving equation system (5), and each $Q_{i j}=\frac{D_{i j}}{L_{i j}}\left(p_{i}-p_{j}\right)$ is also obtained.

It should be noted that the variable $D_{i j}$ evolves according to the adaptation equation (4) while variables such as $p_{i}$ and $Q_{i j}$ are determined by solving the network Poisson equation (5) defined by the $D_{i j}$ 's (and $L_{i j}$ 's) at each instant. As conductivity is closely related to tube thickness, the disappearance of tubes is expressed by zero conductivity of the corresponding tube segment.

We present one simulation outcome in Fig. 2, which shows the process of maze solving by our model. Three groups of dots can be seen in the left upper panel. The left group corresponds to segments of dead-end paths, the right group corresponds to segments that are definitely included in the solution path, and the central group corresponds to segments that are 'competing'. The left group of dots withdraws to the origin, while the right group rises to the top right corner, as shown in the central upper panel. The central group of dots is in the process of differentiating into a 'winning' group and a 'losing' group.

Finally, all the dots (segments) fall into two groups, and the segments belonging to the 'winning' group define the 'solution'. 


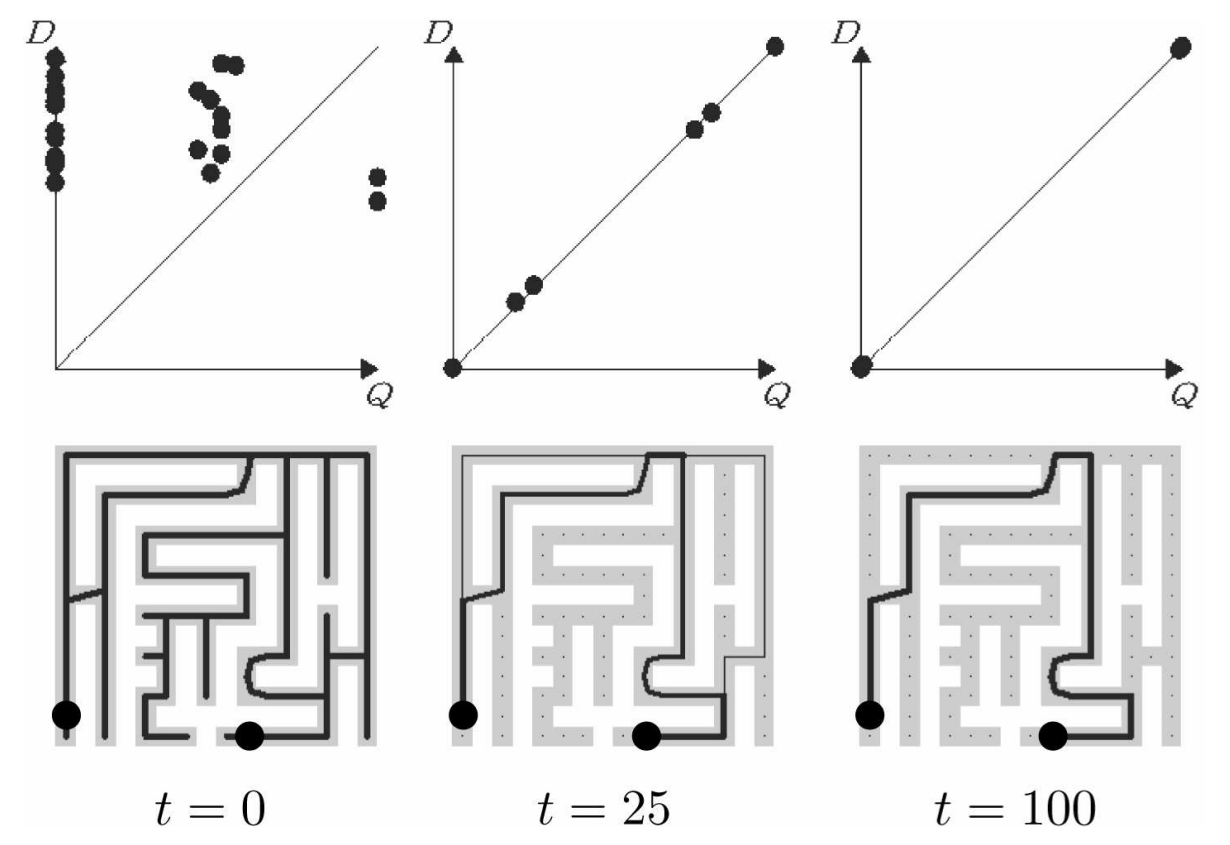

Fig. 2. Sequence of maze solving by Physarum model. The thicknesses of the lines in the lower panels represent the conductivities of the corresponding segments of tube. The dots in the upper panels represent the values of the pair $\left(\left|Q_{i j}\right|, D_{i j}\right)$ of the corresponding segment $M_{i j}$. The parameters are set at $I_{0}=1, \alpha=1$ and $r=1$.

\section{Application to navigation of road map}

We now demonstrate the application of our model to road navigation. Fig. 3(a) ) shows the network of US interstate highways. In this case, we assume that we are planning a car trip from Seattle to Houston using interstate highways and the task is to find the shortest route; the Physarum road navigation system will provide the answer. To do so, it is necessary to possess the node data, in which each node corresponds to a junction in the highway network, and the node connection data that correspond to the distances between connected nodes. Once the data are set by selecting the source node (Seattle) and the sink node (Houston), it is easy to obtain the shortest path using Physarum solver. The result is shown in Fig. 3(d). Fig. 3(b) and (c) show that the more promising paths tend to survive longer in the path finding process.

Suppose that when we drive near Salt Lake City, we are informed that the highway between Oklahoma City and Dallas is unavailable because of an accident, and rapid recovery cannot be expected, as indicated in 4(a). To take this into account, we can again consult the Physarum road-navigation system. We simply need to terminate the path segment corresponding to the unavailable highway, reset the source node to Salt Lake City and run the solver again. This will yield the optimum path at that point, as shown in Fig. 4(b). 

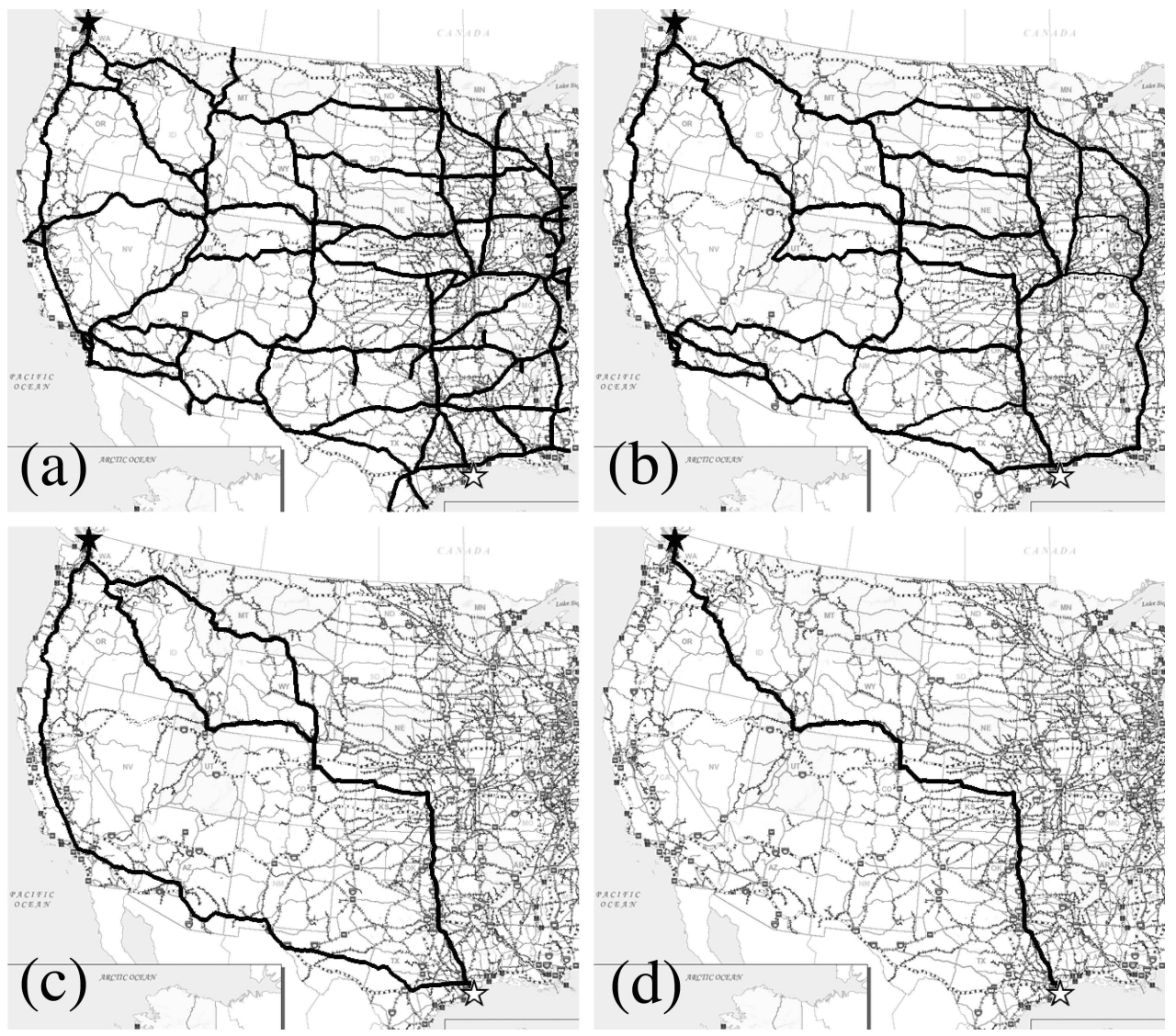

Fig. 3. (a) The network of US interstate highway is expressed by the thick black lines. The starting point (Seattle) and the goal (Houston) are indicated by black and white stars, respectively. (b) and (c) The intermediate stages of the solving process. (d) The shortest path is obtained.
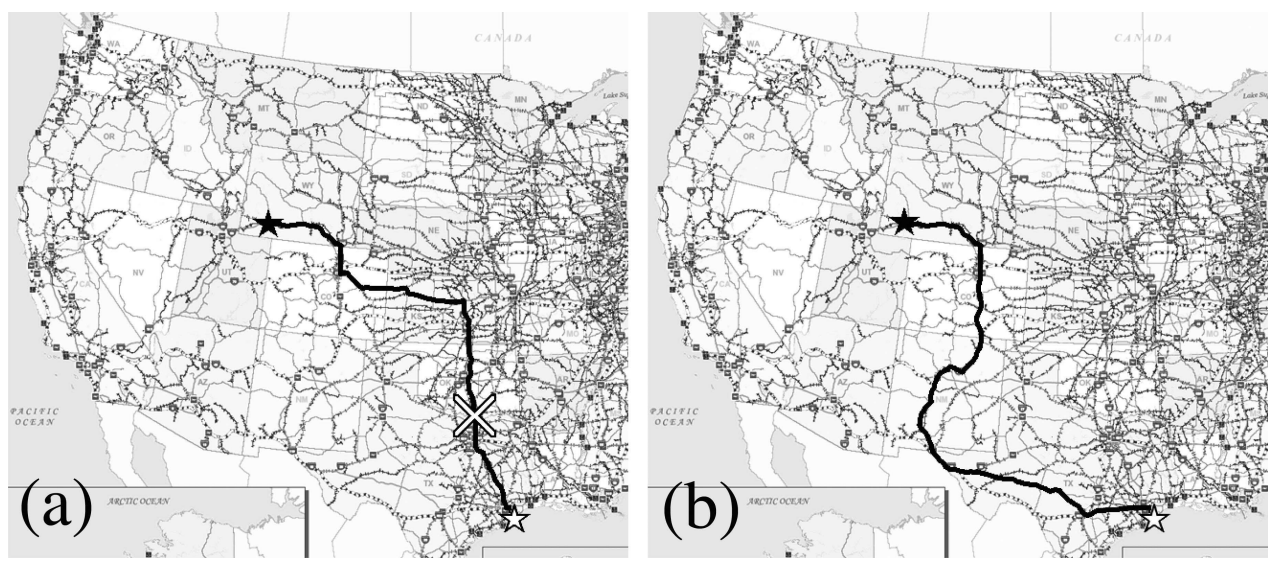

Fig. 4. Dynamic determination of the shortest path. (a) The location of the accident is marked by a white cross. The network and the source (starting) node are reset, maintaining the $D_{i j}$ 's values at that time. (b) The shortest path that bypasses the location of the accident is obtained. 


\section{Summary}

In this article, we have demonstrated a simple mathematical model of pathfinding by Physarum, with the ability to solve even a complex maze. The model reproduces two steps of tube selection: dead-end cutting and selection of the shortest path from among the competing connections. The behaviour of this solver depends on the choice of the form of the function $f(Q)$ : a detailed analysis of this matter will be presented elsewhere.

We have also presented an application to road navigation with relatively straightforward implementation. This is one simple usage of the Physarum system. In future, we will describe further applications to path-finding problems in other networks.

\section{Acknowledgments}

RK is supported by Grant-in-aid for Scientific Research NO. 16654017 of the Japan Society for the Promotion of Science. TN is supported by Grant-in-aid for Scientific Research NO. 15300098 of the Japan Society for the Promotion of Science.

\section{References}

[1] E. Bonabeau, M. Dorigo, G. Theraulaz, Swarm Intelligence, Oxford University Press (1999).

[2] T. Nakagaki, H. Yamada, \& A. Tóth, Maze-solving by an amoeboid organism, Nature 407, 470 (2000).

[3] T. Nakagaki, Smart behavior of true slime mold in labyrinth, Res. Microbiol. 152, 767-770 (2001).

[4] T. Nakagaki, H. Yamada and A. Tóth, Path finding by tube morphogenesis in an amoeboid organism, Biophys. Chem. 92, 47-52 (2001).

[5] T. Nakagaki, H. Yamada and M. Hara, Smart network solution by an amoeboid organism, Biophys. Chem. 107, 1-5 (2004).

[6] T. Nakagaki, R. Kobayashi, Y. Nishiura and T. Ueda, Obtaining multiple separate food sources: behavioural intelligence in the Physarum plasmodium, Proc. R. Soc. Lond. B 271, 2305-2310 (2004).

[7] T. Nakagaki, H. Yamada and T. Ueda, Interaction between cell shape and contraction pattern, Biophys. Chem. 84, 195-204 (2000). 